\title{
El concepto de desarrollo: posiciones teóricas más relevantes
}

\author{
Mujica Chirinos, Norbis* \\ Rincón González, Sorayda**
}

\section{Resumen}

El resurgimiento de la discusión acerca de los modelos de desarrollo se puede ubicar hacia finales de la década de los noventa, cuando se hizo más que evidente que las medidas de ajuste ortodoxas aplicadas en América Latina en particular, no alcanzaron los resultados esperados, lo que generó dudas sobre la concepción y orientación que guiaron estos procesos. Hoy, en el marco de la crisis financiera global y del sistema capitalista en general, el tema vuelve a colocarse en el tapete. En este sentido, el presente artículo tiene como objetivo analizar el concepto de desarrollo desde las posiciones teóricas más relevantes en la segunda mitad del siglo XX y comienzos del XXI. La metodología utilizada para abordar el estudio fue la revisión y análisis teórico, el análisis de contenido y el análisis biblio-hemerográfico, previéndose un horizonte temporal entre 1960 y 2008 , centrándose el análisis en los modelos operacionalizados en este periodo, además de incluir otros enfoques alternativos, dada su importancia teórica y epistémica. Los resultados evidencian que ha existido un marcado énfasis economicista alrededor de la idea de crecimiento económico como expresión de desarrollo, lo que ha motivado algunas respuestas desde otros ángulos. Se concluye en la necesidad de concebir el desarrollo como un proceso altamente complejo, que exige la integración de diversos factores que contribuyan a enriquecer el concepto e impacten las políticas y los actores involucrados en el mismo.

Palabras clave: Desarrollo, concepto de desarrollo, teoría del desarrollo, modelos de desarrollo.

\section{Recibido: 20-01-10. Aceptado: 15-06-10}

* Licenciado en Comunicación Social, mención Periodismo Impreso, de La Universidad del Zulia (LUZ). Especialista en Política Social, Centro de Estudios del Desarrollo (CENDES) de la Universidad Central de Venezuela (UCV). Candidato a Doctor en Ciencias Sociales. LUZ. Investigador del Centro de Estudios de la Empresa y Profesor de la Escuela de Administración y Contaduría Pública y Escuela de Sociología de la FCES, LUZ. Acreditado al Programa de Promoción al Investigador (PPI) Nivel II de la ONCTI. norbism@yahoo.com.

** Socióloga egresada de La Universidad del Zulia (LUZ). Magíster en Planificación y Gerencia de C y T (LUZ). Doctora en Ciencias Gerenciales de la Universidad Rafael Belloso Chacín (URBE). Profesora Titular de la Escuela de Sociología de LUZ. Investigadora del Centro de Estudios de la Empresa, FCES, LUZ. Acreditada al Programa de Promoción del Investigador (PPI) Nivel II de la ONCTI. sorarincon@hotmail.com. 


\title{
The Concept of Development: Most Relevant Theoretical Positions
}

\begin{abstract}
Resurgence of the discussion regarding development models can be located at the end of the 1990s, when it became more evident that the orthodox adjustment measures applied in Latin America in particular, did not achieve the desired results, thereby generating doubts about the concept and orientation that guided these processes. Today, in the context of the crisis of global finances and the capitalist system in general, the theme has been brought up once again. The objective of this article is to analyze the concept of development seen from the most relevant theoretical positions in the second half of the twentieth and beginnings of the twenty-first centuries. The methodology used to approach this study included theoretical review and analysis, content analysis and biblio-hemerographic analysis, focused on the period between 1960 and 2008, centering analysis on the operationalized models of that period, while including other alternative approaches, given the theoretical and epistemological importance of the theme. Results show that a marked economistic emphasis has existed regarding the idea of economic growth as an expression of development, which has motivated some responses from other angles. The study concludes that there is a need to conceive development as a highly complex process, which demands the integration of diverse factors that contribute to enriching the concept and affecting the policies and actors involved in it.
\end{abstract}

Key words: Development, development concept, development theory, development models.

\section{Introducción}

En su Informe sobre el Desarrollo Mundial 1999-2000, titulado En el Umbral del Siglo XXI, el Banco Mundial (BM, 2000) asume una postura autocrítica en cuanto a su concepción del desarrollo, que contrasta con la perspectiva que tradicionalmente había sostenido en informes anteriores, habitualmente centrada alrededor del crecimiento económico, la libertad de mercado y el Estado mínimo.

Este informe aborda una serie de aspectos que colocan la noción de desarrollo en el centro del debate; reconoce que el mismo no puede considerarse úni$\mathrm{co}$, ni alrededor de afirmaciones absolutas, incompletas o dogmáticas, y que existe en su entorno una complejidad determinada por el contexto, el momento histórico y el lugar geográfico.
Plantea, además, que el debate debe abandonar la discusión sobre la función del Estado y el mercado y la búsqueda de soluciones de políticas globales y únicas, para profundizar en otros aspectos más relevantes, como el conseguir mejoras sostenibles en la calidad de vida de las personas.

Para llegar a estas afirmaciones, ejemplifica la experiencia en la aplicación de las políticas de desarrollo a nivel mundial, destacando los resultados disímiles, positivos en algunos países en contraste con los efectos negativos en otros que aplicaron estrategias similares.

Más allá de si se está o no de acuerdo con las afirmaciones anteriores, esta referencia es importante por cuanto, en primer término, en los albores del siglo $X X I$, el propio Banco Mundial, uno de los organismos responsable de la aplicación 
de las medidas de ajuste estructural en América Latina, reconoce que el desarrollo depende de diversos factores, de sus relaciones y de las transformaciones que estos producen en el tiempo.

En segundo término, porque intenta romper con la que, históricamente, ha sido la respuesta a la pregunta sobre qué es el desarrollo, básicamente concebida desde la ciencia económica y ligada a los problemas, argumentos y reflexiones de cada una de las escuelas de pensamiento en esta área a través del tiempo.

Básicamente, se abre un compás de discusión importante que permite abordar el concepto de desarrollo más allá de las posiciones biologicistas de evolución y proceso, y de las tradicionales que lo reducen a problemas de crecimiento económico y redistribución de la riqueza.

En la actualidad, en el marco de la crisis financiera global y del sistema capitalista en general, el tema recibe un nuevo impulso, enmarcado en las respuestas que los organismos internacionales y los gobiernos de países desarrollados impulsan para tratar de superarla, lo que impone una revisión crítica de la experiencia previa y de sus bases analíticas.

En este sentido, el propósito del presente artículo es analizar el concepto de desarrollo según las posiciones teóricas de mayor relevancia durante la segunda mitad del siglo XX y comienzos del $X X I$. La metodología para abordar el objetivo planteado fue la revisión y análisis teórico, el análisis de contenido y el análisis biblio-hemerográfico. Se prevé un horizonte temporal comprendido entre 1960 y 2008; es decir, el estudio se centrará en los modelos operacionalizados en este período -estructuralista y neoliberal-, incluyendo algunas propuestas no aplicadas por su importancia teórica y epistémica -neoestructuralismo, desarrollo sustentable, desarrollo a escala humana, desarrollo humano, enfoque de capacidades-. En absoluto significa que son las únicas; otras propuestas -por ejemplo, el post-desarrollo- no serán abordadas, primero, por motivos de espacio y, segundo, de delimitación teórica, lo cual no significa que carezcan de relevancia.

\section{El estructuralismo y el desarrollo hacia adentro}

El estructuralismo es una corriente teórica de naturaleza económica y social surgida hacia la década de los años 40 , cuyo desarrollo y consolidación estuvo relacionada con la creación de la Comisión Económica para América latina y el Caribe (CEPAL) de las Naciones Unidas y cuyos aportes a la teoría del desarrollo se consideran de vital importancia.

Para los estructuralistas, el concepto de desarrollo es el resultado de la evolución de un conjunto ideas que diferentes tendencias económicas han dado a los problemas específicos del capitalismo y que toma auge a partir de la finalización de la segunda guerra mundial, como preocupación de la Organización de Naciones Unidas por dar respuesta a la situación de los países después del conflicto bélico. Su enriquecimiento conceptual también se asocia a los problemas que se manifestaron desde los países subdesarrollados de América Latina y los nuevos países independientes de África y Asia, que expresaron su inquietud por la excesiva dependencia del comercio mundial 
en tanto su especialización en la exportación de materias primas.

El primer concepto relacionado a la idea del desarrollo para los estructuralistas es el de riqueza; para los autores clásicos -escuela de pensamiento fundada por Adam Smith en el siglo XVIII y consolidada por John Stuart Mill en el XIX- la riqueza es un indicador de la prosperidad o decadencia de las naciones y la consideran como aquel conjunto de bienes que un país puede obtener, dada la naturaleza de su suelo, su clima y su situación respecto a otros países (Sunkel y Paz, 1977; De la Peña, 1979).

Esta concepción de riqueza se relaciona a una determinada manera de percibir la sociedad y su funcionamiento, pensada como un conjunto de individuos o unidades económicas que actúan de acuerdo a leyes y principios inmutables, concepción esta sustentada en la filosofía individualista y liberal del derecho y del Estado difundida en el siglo XVIII, basada en el sistema de libre concurrencia económica y los principios de libertad individual, propiedad privada, sucesión privada de los medios materiales de producción y libertad de contratos.

Así, la riqueza alude a una situación potencial óptima que podría alcanzarse si la sociedad se organiza según un orden individual "natural" que permita el aprovechamiento óptimo de los recursos productivos disponibles.

Posteriormente la idea del desarrollo se asocia al concepto de evolución económica que surge luego de la aparición de las tesis biológicas del evolucionismo darwinista, combinado con el pensamiento del liberalismo económico. De hecho, esta concepción del proceso eco- nómico es básica en las tesis neoclásicas e implica la noción de secuencia natural de cambio, de mutación gradual y espontánea.

Supone la existencia de un orden natural basado en la supervivencia del más fuerte, por lo que la óptima organización social sería aquella que permite crear las condiciones favorables para que opere la selección natural y se garantice la mayor competencia entre los participantes del sistema. Así, la competencia se convierte en la base de sustentación ética ideal para alcanzar la justicia social natural, garantizando la misma a través de la reducción de los factores perturbadores, esencialmente de la intervención del Estado en la economía (De la Peña, 1979).

Muy ligado a la escuela de pensamiento referida anteriormente, se avanza hacia la idea de progreso económico surgida a raíz de la difusión de la revolución industrial y el progreso técnico en Europa y que se resume como la expresión, en el ámbito económico, de la idea de la racionalidad absoluta como posibilidad de funcionamiento humano. Así, en el afán del progreso se adelantaron la ciencia, las artes, las letras y el estilo de vida, teniendo papel significativo las invenciones, los descubrimientos y la afluencia de los metales preciosos provenientes de las colonias, así como también los nuevos productos primarios que se incorporaron al patrón de consumo occidental, por lo que el progreso surgió de un proceso de concentración, explotación colonial y exclusión (De la Peña, 1979; Maza Zavala, 2006a).

Se pensaba que la solución de los problemas sociales se conseguiría a tra- 
vés de la expansión de la producción, lograda gracias a los efectos del permanente cambio científico y tecnológico (De la Peña, 1979). Es decir, lo clave era la modernización a través de la aplicación de la ciencia a las actividades productivas, la incorporación de nuevas técnicas y métodos y la renovación de las estructuras sociales y de las formas de vida.

Cuatro postulados resaltan de esta posición: primero, la irrelevancia de la intervención del Estado en la economía, en tanto la incorporación de la técnica al proceso productivo sea permanente; segundo, la resolución del problema ideológico y la preocupación económica a través de la ciencia; tercero, el problema central de la organización social es administrativo y no político; y cuarto, la operación y funcionamiento de la economía mundial a través de un mecanismo científico, dentro del cual la asignación de los recursos y la distribución geográfica de la actividad, según las capacidades de los países, garanticen el mayor bienestar para todos (De la Peña, 1979).

De allí surge la noción de la división internacional del trabajo basado en las ventajas relativas de cada país, así como la idea del orden mundial, creando espacios económicos que habrían de funcionar con un polo central y una zona de protección en situación de dependencia permanente. Este conjunto de propuestas dan origen a las ideas sobre la superioridad racial europea, sobre el destino manifiesto y sobre el papel rector de los centros imperiales en los acontecimientos mundiales.

De esta forma, el auge del capitalismo estuvo muy ligado alrededor de esta concepción, de tal manera que la innova- ción tecnológica era concebida como motor y fenómeno inherente al sistema, lo que permitía a los economistas neoclásicos teorizar sobre otros elementos -el comportamiento de las unidades económicas individuales; el papel de los mercados y el sistema de precios como instrumento de asignación de los recursos productivos; el papel de las remuneraciones a los factores productivos-, despreocupándose de las leyes de la dinámica del sistema (Sunkel y Paz, 1977; Maza Zavala, 2006a).

En las primeras décadas del siglo $\mathrm{XX}$, se acuña el término crecimiento económico, muy ligado a la teoría macroeconómica y centrada en el interés de preservar la estabilidad, equilibrio y expansión del sistema capitalista y la preservación del status quo mundial. Se pensaba que se había llegado al escalón más alto en la evolución humana, por lo que colocaban su preocupación en los problemas de equilibrio y en el estudio de la crisis -su origen, frecuencia y oportunidad, muy ligado a la preocupación por el desempleo y la ocupación de la mano de obra- para prever su aparición y solucionar las perturbaciones que provocaba (Betancourt, 2004; Bustelo, 1999; De la Peña, 1979; Sunkel y Paz, 1977).

Muy ligado a este enfoque deductivo, existe otra corriente que adopta la vía inductiva para explicar el desarrollo como una sucesión de etapas por la que debe necesariamente atravesar cualquier sociedad y que son las mismas observadas en la evolución de los países desarrollados. Según estas ideas, sostenidas por Rostow y Germani, citados por Maza Zavala (2006a), el subdesarrollo constituiría una fase intermedia en la secuencia o ca- 
mino necesario hacia el desarrollo, concepción esta que motivó esfuerzos importantes en políticas de modernización, entendidas como la racionalización de los valores, actitudes, instituciones y organizaciones de las sociedades desarrolladas (Cf: Sunkel y Paz, 1977; De la Peña, 1979).

Esta posición, que podría plantearse como un progreso en la explicación de las razones del subdesarrollo como un momento en la evolución discontinua de una sociedad, representa un avance con respecto a las teorías de crecimiento, que postula una evolución continua del fenómeno, pero, según Sunkel y Paz (1977) carecen de capacidad analítica para explicar el paso de una etapa a otra; es decir, el proceso de cambio estructural.

Es a partir de este razonamiento que a mediados de este siglo, se comienza a utilizar el concepto de desarrollo surgido del materialismo científico para proponer un cuerpo de doctrina económica en el que predomina la idea de la relación dialéctica de los fenómenos sociales. En su aparición intervienen dos factores: por un lado, la existencia de los países socialistas y, por el otro, el medio hostil -interno y externo- que las naciones más atrasadas enfrentaron en la posguerra. Estos elementos prácticamente quiebran los principios del equilibrio económico de las teorías del crecimiento (De la Peña, 1979).

En su origen, dos corrientes de pensamiento formularon la idea del desarrollo. La primera de ellas fue el desarroIlismo, constituido por partidarios de un capitalismo reformado que proponían la necesidad de una reforma profunda, como salvación del sistema en crisis, ba- sada en la aceleración del desarrollo en los países atrasados a través de la industrialización y, por medio de ella, lograr paliar la presión social con la modificación del comportamiento internacional del mismo (Cf. De la Peña, 1979; Pirela, 1990; Guillén, 2006).

Esta posición, conocida como Estructuralismo, sostenida y planteada por la CEPAL y su fundador Raúl Prebisch, y desarrollada por él y sus colaboradores más cercanos entre los años 40 y 50 del siglo XX -entre ellos Celso Furtado, Anibal Pinto, Jorge Ahumada, Juan Noyola Vásquez, Albert Hisrchman, Aldo Ferrery Osvaldo Sunkel, entre otros- efectúa su análisis del subdesarrollo a partir del concepto centro-periferia, lo que permitió explicar la desigualdad creciente en las relaciones económicas internacionales, así como la heterogeneidad y debilidad estructural de los sistemas productivos y de las estructuras sociales de la periferia. La teoría del deterioro de los términos de intercambio de los productos primarios versus los productos manufacturados, cuestionaba la validez del esquema de división internacional del trabajo que asignaba a la periferia el papel de productor y exportador de productos primarios como mecanismo eficaz para alcanzar el desarrollo (Cf: Guillén, 2006; Maza Zavala, 2006a, 2006b; Furtado, 1996; Pirela, 1990).

La estrategia planteada para superar estos desequilibrios fue conocida como la Industrialización Sustitutiva de Importaciones, originalmente destinada a empoderar a los países subdesarrollados para superar el modelo primario exportador, superar la crisis del modelo de crecimiento hacia afuera y conformar un mer- 
cado interno fuerte como principal dinamizador de la actividad económica; es decir, crear un modelo de desarrollo hacia adentro o desarrollo endógeno (Cf: Sunkel y Paz, 1977; Furtado, 1996; Iglesias, 1993; Maza Zavala, 2006b)

La segunda corriente es la marxista, basada en la necesidad de controlar y racionalizar la evolución de la economía en un contexto social y político apropiado con miras al establecimiento del socialismo como meta final. Según su análisis, en la consecución de este objetivo puede colaborar el cambio evolutivo -y no sólo el revolucionario-, por lo que el desarrollismo supone reformas que se acercarían a esta meta (Cf. De la Peña, 1979).

Esta corriente, surgida de la influencia del Estructuralismo Cepalino, pone énfasis en reformas estructurales, en el papel del Estado como guía, promotor y planificador del proceso, y en la transformación de las modalidades de financiamiento externo y del comercio internacional. Planteaba, además, la definición de un marco metodológico adecuado para el abordaje del fenómeno que permitiera enfocarlo desde un punto de vista estructural, histórico y totalizante, centrado en el análisis y la explicación y no en la descripción (Sunkel Paz, 1977).

En este marco, el desarrollo y subdesarrollo debían entenderse como un sistema único, de estructuras parciales, pero interdependientes, diferenciada una de otra en que la primera, dada su capacidad endógena de crecimiento, es dominante, y la segunda, en función del carácter inducido de su dinámica, es dependiente. En este sentido, el problema fundamental de una estructura subdesarrollada es la necesidad de superar el estado de dependencia, transformando su estructura para obtener mayor capacidad autónoma de crecimiento y reorientar su sistema económico a la satisfacción de los objetivos de la sociedad en su conjunto.

Así, el desarrollo es concebido como un proceso de cambio social, deliberado, cuyo objetivo último es la igualación de oportunidades sociales, políticas y económicas, tanto en el plano nacional como en las relaciones con otras sociedades más avanzadas, que coloca el acento en la acción, en los instrumentos de poder político y en las propias estructuras de poder para la orientación, eficacia, intensidad y naturaleza del cambio. Esta orientación analítica fue conocida como la Teoría de la Dependencia o la Ciencia Social Latinoamericana, donde destacaron intelectuales como Fernando Henrique Cardoso, Theotonio Dos Santos, Vania Bambirra, André Gunder Frank y Enzo Felletto, entre otros (Cf: Sunkel y Paz, 1977; Pirela, 1990; Guillén, 2006).

De esta idea del desarrollo se desprende toda la elaboración teórica que predominó en las décadas que van desde los años cincuenta hasta finales de los ochenta. Posteriormente, luego del agotamiento del estructuralismo-desarrollismo, evidenciado por la crisis de la deuda externa y reforzada por el derrumbe del socialismo real y el proceso de globalización, resurgirán las ideas liberales renovadas con el prefijo nuevo, dando origen al nuevo liberalismo, neoliberalismo o pensamiento único. Así mismo, daría pie a la búsqueda de alternativas diferentes, que intentan superar el reduccionismo economicista característico de la nueva 
posición, intentando incorporar la preocupación por los problemas sociales y ambientales surgidos como consecuencia de la implementación de ambos modelos.

\section{El neoliberalismo y el desarrollo como crecimiento}

La raíz y el origen del Neoliberalismo y su concepción del desarrollo, tal como sugiere su propio nombre, deben buscarse en el liberalismo, aunque su auge en la región Latinoamericana en las décadas de los ochenta y noventa, estuvo marcada por lo que se conoció como Consenso de Washington, suerte de recetario preparado por el Banco Mundial, Fondo Monetario Internacional y la Secretaría del Tesoro de Estados Unidos como instrumentos de política económica que los gobiernos del área debían ejecutar para garantizar el pago de los compromisos económicos y poder acceder al financiamiento luego que hiciera crisis el endeudamiento externo.

El antecedente más remoto, entonces, está en la escuela clásica, fundada por Adam Smith, y la publicación de sus obras Teoría de los Sentimientos Morales y Una Investigación de las Causas y la Naturaleza de la Riqueza de las Naciones, y que cierra con la obra de J. S. Mill. Como afirmamos anteriormente, el interés fundamental de los clásicos se centró en la preocupación por el crecimiento a largo plazo: sus causas, consecuencias y perspectivas. Consideraban que era la acumulación de capital (la reinversión del excedente) el motor principal del crecimiento y de la riqueza de las naciones (Betancourt, 2004; Bustelo, 1999; Sunkel y Paz, 1977).
En este sentido, la idea de mercado y el óptimo resultado estaban en el centro de su análisis como el núcleo del sistema económico, que operaba mediante ensayo y error. Para los clásicos, las decisiones particulares de los individuos se orientan por datos que ofrece el mercado y son moldeadas por acercamientos sucesivos a éste. En estas decisiones, si bien el individuo obra por su propio interés (homo economicus), contribuye a moldear la cantidad y tipo de bienes que se necesita producir, operando un dispositivo propio al sistema económico -la mano invisible- que actúa como orden y ley natural en el aparente desorden de la vida económica; es decir opera un mecanismo inherente a su propia naturaleza, por lo que sus resultados, si no existen trabas institucionales que impidan la decisión libre de los individuos para obtener una ventaja máxima, son óptimos, orientando de este modo cuánto, cuándo y cómo producir (Sunkel y Paz, 1977; Bustelo, 1999; Betancourt, 2004; Maza Zavala, 2006a).

El mercado, a su vez, fomenta la división del trabajo, lo que tiene influencia en la productividad de la mano de obra; esta especialización conlleva a una mayor destreza del obrero, ahorro de tiempo y a la innovación técnica para facilitar y abreviar el trabajo, además de reducir la labor de muchos hombres a pocos. Los aumentos de productividad, aunado los incrementos en la inversión, trae como consecuencia un aumento de la producción total y por habitante. De esta manera, los clásicos ven como principal factor del crecimiento la acumulación de capital, de la que dependen la ampliación del mercado, el grado de división social del 
trabajo y el aumento de los salarios, que permiten un aumento de la renta nacional y de la oferta de la mano de obra (Betancourt, 2004; Bustelo, 1999; CLAT, 1993; Sunkel y Paz, 1977).

Esta es, en síntesis, lo básico del pensamiento clásico y plataforma sobre la cual se desarrollan las propuestas posteriores que se conocen con el nombre de neoclásicos, cuya hegemonía va desde el decenio de 1870 hasta la aparición del Keynesianismo en los años 30 del siglo $\mathrm{XX}$.

En términos concretos, la escuela neoclásica poco aporta a la base analítica de la que parte; su interés fundamental está en el desarrollo de instrumentos de análisis cuyo objetivo es elaborar aspectos parciales de la concepción clásica y explicarla en forma más satisfactoria. Así, se desentendieron del interés en el crecimiento en aras de una preocupación casi exclusiva por cuestiones estáticas y a corto plazo (Betancourt, 2004; Anderson, 2000; Bustelo, 1999; Gómez, 1998; CLAT, 1994).

Fundamentalmente, el interés de los neoclásicos se desplazó hacia el análisis microeconómico y temas en la esfera de la circulación: asignación de recursos, intercambio de mercancías y distribución de la renta. También sustituyeron la teoría clásica del valor-trabajo por una nueva aproximación subjetiva al valor basada en la utilidad y la escasez, así como una teoría de la distribución fundamentada en las productividades marginales de los factores y una función del ahorro determinada por el tipo de interés, distinta a la vinculación clásica y marxista entre distribución y ahorro, dando origen la economía matemática (Betancourt, 2004; Anderson, 2000; Bustelo, 1999; CLAT, 1994).

Otra cuestión puntual de los neoclásicos fue el modelo de competencia perfecta como única posibilidad de aproximación teórica a la realidad, en tanto estructura lógica que pretende captar lo esencial de la misma, y que buscaba reforzar el teorema de la mano invisible.

Este modelo supone la existencia de agentes económicos racionales que tratan siempre de maximizar o minimizar una función objetivo, bien sea esta el beneficio, la utilidad o la productividad. Presume también que los costos de información son cero; es decir, que dichos agentes tienen pleno conocimiento sobre las variables y procesos que manejan, asumiendo también que los precios son flexibles tanto al alza como a la baja y que los factores de producción tienen libertad para ir de una ocupación a otra o de un sector de la economía a otro. Asume también que los costos de transacción son cero, que tanto la oferta como la demanda están perfectamente atomizadas ${ }^{1}$ y que está excluido el más mínimo uso del poder o fuerza que pudiesen tener los agentes económicos. Si todos estos supuestos se validan en una economía $X$,

1 Se entiende por Atomizada, un número de oferentes y demandantes tal, que ninguno de ellos pueda influir sobre los precios ni sobre ninguna otra variable que opera en el mercado (Gómez, 1998). 
entonces el precio será único, la oferta y demanda estarán en equilibrio, el costo marginal se igualará al ingreso marginal y éste al precio, la ganancia llegará al máximo y la marginal será cero, el ahorro igualará a la inversión equilibrando la tasa de interés, la demanda de trabajo será igual a la oferta -se estará en pleno empleo-y la cantidad de bienes y servicios producidos será la más elevada posible, disponible equitativamente para todos los que participan en el proceso (Cf: Betancourt, 2004; Bustelo, 1999; Gómez, 1998).

En otras palabras, el análisis microeconómico tenía como objetivo presentar una teoría del consumidor que partía del individualismo metodológico (comportamiento social como la suma de acciones individuales) y defendía el comportamiento racional del consumidor en tanto Homo economicus que busca maximizar su ventaja. En este sentido, la ruptura con la tradición clásica es evidente: sesgo atomista frente al enfoque holista y soberanía del consumidor frente a la primacía de la oferta (Anderson, 2000; Bustelo, 1999; Gómez, 1998).

Por otra parte, el análisis marginalista permitió defender planteamientos sencillos, expresivos y susceptibles de ser modelizados: el consumidor aumenta su demanda hasta que la utilidad marginal se anula, el salario iguala a la productividad marginal del trabajo, las empresas maximizan sus beneficios cuando el ingreso marginal es igual al costo marginal (Anderson, 2000; Bustelo, 1999; Gómez, 1998).

Igualmente, el libre juego de las fuerzas de oferta y demanda (la lógica perfecta del mercado) tiende a establecer, en condiciones de competencia, precios de equilibrio que garantizan una asignación óptima de recursos. Esa creencia en las virtudes del mercado para conducir la economía hacia el equilibrio espontáneo y estático excluía la dinámica y la dimensión histórica de los procesos económicos (Anderson, 2000; Bustelo, 1999; Gómez, 1998).

En este sentido, la crítica a la tradición neoclásica viene dada, entonces, por el abandono de la preocupación clásica y marxista por el crecimiento, por no considerar fenómenos colectivos y las preferencias no racionales en su análisis microeconómico, por su extrema confianza en la lógica del mercado que conduce a no considerar disfunciones o fallas del mismo, porque su teoría de la distribución basada en las productividades marginales se encuentra aislada de las relaciones sociopolíticas y se ve reducida a la simple formación de precios, y por desatender las políticas de demanda, necesarias para el mantenimiento del empleo -aceptación de la Ley de Say² - (Bustelo, 1999; CLAT, 1994). mica que se conoce como la Ley de Say, según la cual "toda oferta crea su propia demanda", lo que significa que al producir bienes y servicios hay que remunerar a los factores productivos, exactamente en la misma cantidad de valor creada en dicho proceso y lanzada al mercado, generando así al menos la capacidad de compra necesaria para demandar la masa de bienes 
En resumen, podemos afirmar que no existió explícitamente una teoría neoclásica del desarrollo, puesto que la insistencia en el equilibrio espontáneo entre oferta y demanda en los mercados eliminaba la variable tiempo del análisis. Sin embargo, implícitamente, se asumía una concepción de desarrollo como proceso gradual, continuo, armónico y acumulativo de crecimiento; gradual, influenciado por las tesis darwinistas de la evolución social; continuo porque la naturaleza económica, particularmente la innovación y difusión técnica, carecían de fisuras; armónico, porque beneficia a todos los perceptores de renta-el mercado genera por sí mismo tendencia al pleno empleo y al aumento de los salarios reales-; y acumulativo, pues el crecimiento se extiende, como una mancha de aceite, entre unos y otros sectores (Bustelo, 1999; CLAT, 1994).

\section{El neoestructuralismo y el desarrollo desde dentro}

La renovación del pensamiento estructuralista-Cepalino se produce a raíz de los resultados adversos que la aplicación del Consenso de Washington produjo en la región Latinoamericana, en el rechazo a tales políticas y en la necesidad de recuperar la senda del desarrollo para superar lo que se conoció como la década perdida.

El neoestructuralismo se nutre, principalmente, del pensamiento estructuralista convencional, intentando corre- gir, formalizar e integrar algunos supuestos para hacerlos más rigurosos en el análisis macroeconómico.

El neoestructuralismo surgió como otra vertiente teórica distinta del enfoque ortodoxo neoliberal del ajuste, intentando aportar soluciones por la vía de los programas de estabilización y de ajuste heterodoxos de los años ochenta (...) Pero en la medida que muchos de los planes de ajuste de una y otra característica fracasaban y la crisis persistía, el neoestructuralismo comenzó a recurrir y a nutrirse del legado positivo de un ideario propiamente latinoamericano sobre desarrollo: el estructuralismo (...) (El neoestructuralismo) pretende contribuir al enriquecimiento y puesta al día de dicha línea de pensamiento, concentrando el esfuerzo intelectual tanto en el bosquejo de una estrategia renovada de desarrollo "desde dentro", en su dimensión global, como en la elaboración operativa macro, micro y meso económicas de carácter selectivo (Ramos y Sunkel, 1995:17-18).

En este marco, mientras el neoliberalismo se caracteriza por una visión individualista, utilitaria y ahistórica (Homo economicus), el neoestructuralismo parte de un enfoque sociocultural e histórico (Homo sociologicus), nutriéndose, con eclecticismo postmodernista, de todas las disciplinas científicas y corrientes de pensamiento capaces de aportar elementos relevantes, incluyendo la teoría neoclásica.

cuya producción ha creado dicha capacidad de compra. Esta es la estructura esencial sobre la que se sustenta el mercado (Gómez, 1998). 
(El Neoestructuralismo) interpreta el comportamiento económico de los agentes individuales según contextos históricos, de carácter socioeconómico e institucional, en que tales agentes formulan sus opciones y desarrollan sus conductas. Considera que los individuos se estructuran en grupos sociales organizados en una multiplicidad de instituciones públicas y privadas, las que desarrollan con el tiempo un conjunto de valores y reglas de comportamiento. Estas formas de organización social constituyen a su vez verdaderas culturas que delimitan y orientan las conductas individuales (Sunkel, 1994:31-32).

A partir de estos planteamientos, el pensamiento Neoestructural se nutre de dos pensadores y teóricos: Fernando Fajnzylber y Osvaldo Sunkel, cuyas propuestas se sintetizarán posteriormente en el documento de la CEPAL Transformación Productiva con Equidad. La Tarea Prioritaria para América Latina y el Caribe en los Años Noventa (Cepal, 1990). Luego, estas ideas se profundizarán y complementarán en los documentos El Desarrollo Sustentable: Transformación Productiva, Equidad y Medio Ambiente (Cepal, 1991), Educación y Conocimiento: Eje de la Transformación Productiva con Equidad (Cepal, 1992) y Equidad y Transformación Productiva:
Un Enfoque Integrado (Cepal, 1996), cerrando este corolario Equidad, Desarrollo y Ciudadanía (Cepal, 2000), donde la propuesta Neoestructural alcanza su mayor configuración teórica ${ }^{3}$.

Los aportes centrales de Fernando Fajnzylber pueden dividirse en dos aspectos: por un lado la teoría de la Caja Negra del progreso técnico y, por el otro, la del Casillero Vacío del dinamismo con equidad (Fajnzylber, 1983, 1990; Bustelo, 1999; Fernández-Muro, 2004; Olivé, 2004; Torres, 2006).

Para llegar a estos conceptos, Fajnzylber hace un análisis de la experiencia de industrialización en América Latina centrada en la crítica del enfoque neoliberal acerca de las insuficiencias de este proceso en la región. Reconociendo el patrón industrial precedente como trunco y distorsionado, referido al grado de industrialización más que a su contenido, propone una nueva industrialización basada en la ampliación de las alianzas sociales que sustentaban el patrón anterior, desplazando el centro de interés hacia los sectores sociales mayoritarios. No se trata de fomentar las exportaciones en lugar de sustituir importaciones; el objetivo es construir un núcleo endógeno capaz de incorporarse en el proceso de dinamización tecnológica como condición para penetrar y mantenerse en el mercado internacional en vez de delegar en agentes

3 Es importante reconocer el aporte al neoestructuralismo realizado por el economista Lance Taylor de la Universidad de Harvard, el Instituto Tecnológico de Massachusetts (MIT) y del New School for Social Research en los Estados Unidos. Básicamente, los estudios de Taylor están enfocados al interés, desde el punto de vista normativo, en las políticas de desarrollo para desmontar, desde dentro, las recetas ortodoxas y la adaptación de las políticas a las circunstancias particulares de cada país (Fernández-Muro; 2004; Bustelo, 1999). 
externos la definición de la estructura productiva del país.

El concepto de Caja Negra es producto del análisis que realiza, en primer lugar, del vínculo progreso técnico-manufactura, señalando que los esfuerzos de innovación y desarrollo tecnológico no se distribuyen homogéneamente en el conjunto de la actividad productora, sino que están concentrados en el sector manufacturero. En los países industrializados la producción industrial representa entre un cuarto y un tercio del PIB y los gastos de investigación y desarrollo absorben más del $90 \%$ de los recursos, lo que explica el mayor dinamismo de la demanda de estos productos en comparación con los recursos naturales y su influencia en la relación de precios del intercambio entre ambos sectores.

También, dentro del sector manufacturero existen ramas donde se concentra el esfuerzo tecnológico, básicamente aquellas que han experimentado mayor crecimiento de la posguerra y que registran mayor dinamismo en el comercio internacional, por lo que es necesario identificar y aislar los sectores que tiene mayor responsabilidad en ellos. Es decir, es clave el papel de la desagregación sectorial, asociado al crecimiento de la productividad total de factores y cuya expansión está determinada por el progreso técnico, ya que hay ramas manufactureras que son portadoras de mayor contenido tecnológico que otras.

En segundo lugar, el papel de los aspectos micro o sectoriales, en contraposición y crítica al análisis macroeconómico tradicional de crecimiento centrado en indicadores de corto plazo, sustentado por la corriente neoliberal, para la cual la desagregación sectorial es inservible para alcanzar sus propósitos analíticos relacionados con los equilibrios macroeconómicos. Precisamente, la superación de esta deficiencia se expresa en la necesidad de avanzar en la desagregación sectorial para abrir la Caja Negra del progreso técnico.

En Tanto, el Casillero Vacío pone en evidencia el hecho de que la gran mayoría de los países de la región Latinoamericana muestra patrones de crecimiento incompatibles con la equidad. Utilizando un diagrama de cuatro casilleros, donde cruza dos ejes -uno correspondiente al crecimiento económico medio de los países industrializados entre 1965-1984, y el otro a un indicador de equidad en la distribución del ingreso entre 1970-1984- demuestra que del total de países de América Latina, varios registran tasas de crecimiento por debajo de la media y estándares de equidad regresivos, mientras otros crecen por encima del promedio, pero su estándar distributivo es todavía más desigual que la media de los países industrializados; además, evidencia que ninguna economía Latinoamericana crece con equidad. Es precisamente esta última constatación empírica la que Fajnzylber llama el síndrome del Casillero Vacío.

Parecería así que el rasgo central del proceso de desarrollo Latinoamericano es la insuficiente incorporación de progreso técnico, su escasa aportación de pensamiento original basado en la realidad para definir el abanico de decisiones que supone la transformación económica y social. El casillero vacío estaría vinculado directamente con lo que podría llamarse la incapacidad para 
abrir la 'caja negra' del progreso técni$\mathrm{co}$, tema en el que incide el origen de las sociedades latinoamericanas, su institucionalidad, el contexto cultural y un conjunto de factores económicos y estructurales, cuya vinculación con el medio sociopolítico es compleja, pero indiscutible (Fajnzylber, 1990:4).

En el caso de Sunkel (1995), propone avanzar del desarrollo hacia adentro de la estrategia estructuralista y el desarrollo hacia afuera de los neoliberales, al desarrollo desde dentro como eje central de la propuesta Neoestructural. Este planteamiento lo ubica en los estudios de Prebisch:

Resulta claro que en su planteamiento original (Prebisch) distinguía ambas etapas en términos de compensar el estímulo dinámico de la propagación de la técnica que provenía 'desde afuera', y que se había hecho insuficiente, mediante el desarrollo de dicho estímulo, 'desde dentro'. El cambio de adverbio sugiere una distinción fundamental. Prebisch pensaba en un proceso interno de industrialización capaz de crear un mecanismo endógeno de acumulación y generación de progreso técnico y mejoras de productividad como el que se construyó a partir de la Revolución Industrial en los países centrales (Sunkel, 1995:63).

En el fondo, se trataba de asimilary no de transferir, copiar o reproducir el progreso técnico, colocando el acento en los modos de producir; es decir, de la oferta. Sin embargo, el desarrollo hacia adentro colocó el énfasis en la demanda, conduciendo a una estrategia basada en la ampliación del mercado interno que reprodujo los patrones de consumo y producción de los centros, apoyada por la sustitución de importaciones, y orientó una demanda interna estrecha y sesgada, lo que configuró un patrón de distribución desigual del ingreso (Sunkel, 1995; Bustelo, 1999; Fernández-Muro, 2004; Olivé, 2004).

Para Sunkel (1995), el desarrollo desde dentro supone implicaciones diferentes, cuyas bases, planteadas por Fajnzylber, estiman, primero, un esfuerzo por configurar una estructura productiva funcional, estableciendo industrias fundamentales para crear y potenciar un núcleo endógeno básico que lidere el nuevo proceso de industrialización, acumulación, generación y difusión del progreso técnico e incremento de la productividad. Segundo, posterior a esta etapa fundacional, se requiere reforzar la creatividad interna a través de la interrelación y participación de los diversos actores y agentes socio-productivos. Logrado este nivel de interacción e integración en la práctica, se conformaría en términos reales el concepto de núcleo endógeno.

Sin embargo, el planteamiento anterior estaría ligado al paso de un ajuste recesivo de orientación neoliberal a un reajuste expansivo que garantice, una transición hacia el desarrollo, la consolidación de la democracia y la superación de la pobreza.

En este sentido, la diferencia entre ajuste recesivo y reajuste expansivo es clave. Si bien el primero busca liberar recursos para el pago de la deuda externa, el reajuste expansivo tiene como objetivo el pago de la deuda social, dando prioridad tanto a las acciones de corto plazo como las de largo plazo destinadas a reducir la extensión e intensidad de la pobreza mediante cambios distributivos ne- 
cesarios para alcanzar un nivel de equidad socialmente aceptable, combinando una política restrictiva selectiva de la demanda con una política selectiva de expansión de la oferta para lograr su ajuste recíproco (Sunkel, 1995; Bustelo, 1999).

De forma tal que el neoestructuralismo, si bien asume el concepto de desarrollo propuesto por el estructuralismo como un proceso deliberado de cambio social, cuyo objetivo último es la igualación de oportunidades sociales, políticas y económicas, agrega la necesidad de rellenar el "casillero vacío" del dinamismo y crecimiento con equidad, a través del logro de la competitividad internacional basada en el progreso técnico y la incorporación de valor intelectual a los recursos naturales, lo que impone la necesidad de abrir la "caja negra" del progreso técnico para el logro del círculo virtuoso de la equidad y la competitividad.

El logro de la equidad, la justicia social y la profundización democrática, estarían ligados al paso de un desarrollo hacia adentro -estructuralista- y hacia afuera-neoliberal-a un desarrollo desde dentro, en torno a un proceso endógeno de acumulación, absorción y generación de progreso técnico que oriente un nuevo proceso de industrialización basado en la oferta y orientado no sólo a la demanda interna, sino también hacia la exportación.

\section{El desarrollo sustentable}

La incorporación de la preocupación medioambiental a la agenda de desarrollo se puede considerar de reciente data, liderizada por la Organización de las Naciones Unidas. Distintos autores
(Reed, 1996; Jiménez Herrero, 1997; De Lisio, 1999) ubican los antecedentes más inmediatos en la Conferencia de las $\mathrm{Na}$ ciones Unidas sobre el Entorno Humano, celebrada en Estocolmo en 1972, aunque otros (Gabaldón, 2006) lo ubican a finales del siglo XIX y comienzos del XX, producto de un proceso donde confluyeron la incipiente ciencia ecológica, la conservación de los recursos naturales y la protección del medio ambiente, que atrajo la atención de profesionales de distintas disciplinas y de integrantes de la sociedad civil.

Su conformación teórica fue producto, en primer término, de las presiones y exigencias de movimientos civiles a nivel mundial para que los gobiernos enfrentaran las crecientes crisis ambientales y, en segundo término, de las tensiones existentes entre las perspectivas de desarrollo del norte industrializado y el sur en desarrollo (Reed, 1996; Jiménez Herrero, 1997; De Lisio, 1999).

A ella contribuyeron, además de la reunión de Estocolmo, la Conferencia de las Naciones Unidas para el Ambiente y Desarrollo (UNCED) celebrada en Río de Janeiro en 1992, conocida también como la Cumbre de la Tierra y como Eco 92; así mismo, la Estrategia para la Conservación Mundial -publicada por la Unión Internacional para la Conservación de la Naturaleza y de los Recursos Naturales (UICIN), el Programa de las Naciones Unidas para el Ambiente (UNEP) y el Fondo Mundial para la Naturaleza (WWF) en 1980-, y el informe ofrecido por la Comisión Mundial para el Ambiente y el Desarrollo (WCED) en 1987, conocida como la Comisión Brundtland (Cf. Reed, 1996; Jiménez Herrero, 1997). 
Dichas reuniones e informes aportaron elementos importantes para la conformación del enfoque del Desarrollo Sustentable: la aceptación de que la contaminación ocasionada por la industrialización de los países del norte imponía restricciones al avance de los países en desarrollo; la aceptación del enfoque del sur de que era la pobreza, y no la industrialización, la causa de los problemas ambientales del mundo en desarrollo para los cuales el crecimiento económico debía ofrecer la respuesta principal; la vinculación del bienestar humano actual y futuro con la administración sostenible del patrimonio natural del planeta; el establecimiento de la responsabilidad de la actual generación con respecto a la protección de los recursos naturales y el ambiente para garantizar las oportunidades de desarrollo de las futuras generaciones; $y$ el reconocimiento de la necesidad de reordenar las estructuras del comercio internacional y de los flujos de capital para asegurar mayor influencia de los países en desarrollo en estas relaciones, dando nueva forma a la búsqueda de la sustentabilidad en el marco de la economía global (Reed, 1996; Jiménez Herrero, 1997; De Lisio, 1999).

Si bien los logros de estos eventos, especialmente Eco 92, fueron considerables, los mismos no alteraron las condiciones políticas que facilitaran el transito de las naciones del mundo hacia la nueva estrategia, ni modificaron las concepciones tradicionales del desarrollo como crecimiento económico, ni incluyeron, además de la económica y ambiental, la dimensión social dentro del esquema global de discusión sobre la sustentabilidad.
Sin embargo, es importante rescatar que sus fundamentos conceptuales se han enriquecido por un esfuerzo intelectual inter y transdisciplinario, además de provocar respuestas que tratan de cambiar la perspectiva estratégica tradicional de estas agencias -de arriba hacia abajo- por otra más ligada a los protagonistas y actores -de abajo hacia arriba-, como es el caso de el Enfoque Operacional del Desarrollo Sostenible, auspiciado por el Fondo Mundial para la Naturaleza (WWF), la Unión Internacional par la Conservación de la Naturaleza (IUNC) y el Programa de las Naciones Unidas para el Medio Ambiente (UNAMP) (Cf. Reed, 1996; Jiménez Herrero, 1997; De Lisio,1999).

Así, el punto común que comparten los distintos autores se toma básicamente del informe Brundtland de 1987, que define el Desarrollo sustentable como un curso humano capaz de satisfacer las necesidades y aspiraciones de la generación presente, sin comprometer las posibilidades y habilidades de las generaciones futuras de satisfacer las suyas (Reed, 1996; Jiménez Herrero, 1997; De Lisio, 1999; Castellano, 2005; Gabaldón, 2006). Es decir,

El desarrollo (sustentable) tiene como punto central la gente, en el sentido de que su principal objetivo es el mejoramiento de la calidad de vida del hombre, y está fundamentada en la conservación en el sentido de estar condicionado por la necesidad de respetar la capacidad de la naturaleza para el suministro de recursos y servicios para el mantenimiento de la vida. Bajo este punto de vista, el desarrollo (sustentable) significa el mejoramiento de la calidad de vida 
del hombre mientras se mantenga dentro de la capacidad de apoyo de los ecosistemas de soporte (Reed, 1996: 48).

Más recientemente, Meadows et al. (1992) y Gladwin y Krause (1995), citados por Castellano (2005), amplían esta la definición; en el primer caso, plantean que el desarrollo sustentable es aquel que puede persistir a lo largo de generaciones, ve suficientemente hacia adelante, es suficientemente flexible y suficientemente sabio como para no socavar sus sistemas de soporte físico o social.

En el segundo caso, definen el desarrollo sustentable como el proceso de lograr el desarrollo humano de manera incluyente -en el tiempo y el espacio-, conectada-interdependencia de lo ecológico, lo económico y lo social-, equitativa -justicia intergerenacional, intrageneracional e interespecies-, prudente -deberes de cuidado y prevención tecnológica, científica y política-y segura -estar a salvo de amenazas crónicas y protección contra disrupciones dañinas-.

Tal y como se desprende, subyacen tres componentes básicos dentro de la definición de desarrollo sustentable: uno económico, uno social y otro ambiental. El económico, expresa la exigencia de que las sociedades se encaminen por sendas de crecimiento económico sostenible que genere un real aumento del ingreso y mejore la distribución; la dimensión social presupone la equidad -asegurar el acceso de todas las personas a niveles mínimos de salud, educación, seguridad, vivienda, etc.- y la interdependencia de las comunidades humanas como requisito para una calidad de vida aceptable; el componente ambiental se basa en el mantenimiento de la integridad y la productividad a largo plazo de los sistemas que mantienen la infraestructura ambiental y la vida del planeta (Cf: Reed, 1996; Jiménez Herrero, 1997).

\section{El desarrollo a escala humana}

Como hemos observado hasta el momento, existe un sesgo marcadamente economicista en la concepción y el tratamiento de los elementos que conforman el concepto de desarrollo. Aunque el neoestructuralismo introduce su preocupación por el tema de la equidad, el mismo aparece muy ligado al problema del progreso técnico que genere crecimiento sostenido acompañado de políticas redistributivas; así mismo, el desarrollo sustentable incorpora un componente social en sus dimensiones, pero se percibe subsidiario de lo económico y ambiental, aunque el logro de la sustentabilidad radicaría en el equilibrio de los tres.

Una respuesta a estos enfoques la conseguimos en Manfred Max-Neef (1986a; 1986b) y su propuesta de Desarrollo a Escala Humana. Podemos afirmar que su origen está muy ligado a la crisis y contradicciones personales-profesionales del autor con respecto a la manera cómo la economía se fue desligando de su interés central: el bienestar de la gente. Básicamente, la crítica proviene de la manera mecanicista en que la ciencia económica ha concebido y abordado los problemas del hombre.

La economía, que originalmente se derivó de la filosofía moral, perdió de pronto gran parte de su dimensión humana que fue reemplazada por teorías caprichosas y trivialidades técnicas, incom- 
prensibles para la mayoría e inútiles para todos, excepto tal vez para sus autores que suelen ganar premios por haberlas elaborado (...) Como disciplina (...) se ha convertido repentinamente en una de las materias más importantes de la actualidad. No habría nada de malo en ello si la importancia dada a la ciencia económica correspondiera realmente a su capacidad de interpretar y resolver los problemas que afectan a la humanidad. Este no es el caso (MaxNeef, 1986a: 23 y 37).

Este conflicto lo lleva a identificar cuatro áreas críticas desde donde arma su propuesta: la admiración ilimitada por el gigantismo y las grandes soluciones; la obsesión por las mediciones y cuantificaciones; el enfoque mecanicista para la solución de los problemas económicos; y la tendencia a simplificar en exceso, que se refleja en la preferencia por la objetividad técnica a costa de la pérdida de una visión moral, del sentido de la historia y la inquietud por la complejidad social (MaxNeef, 1986a).

En lo fundamental, Max-Neef (1986a) considera que los modelos de desarrollo economicistas, han desplegado una excesiva admiración hacia lo macro y lo gigante de los sistemas que lo integran, en términos tanto de producción como de distribución, ligado a la eficiencia, sin importar los efectos adversos que puedan tener en el bienestar de la gente que participa en ellos y en su entorno físico-natural-social. Así mismo, han definido sistemas de mediciones selectivas y discriminatorias cuando se refieren a los seres humanos (producto interno bruto, sistema de precios, tasas de crecimiento, etc.), que ocultan tareas y actividades realizadas a nivel doméstico, por lo que la mayoría de las personas son estadísticamente invisibles en términos económicos.

Si bien considera al crecimiento económico como positivo, también lo cree insuficiente, además que el mismo oculta, solapa y se equipara al concepto de justicia social bajo el sistema de acumulación capitalista; es decir, la justicia social se confunde con el crecimiento, gracias a la búsqueda obsesiva de aumentos significativos del PIB interpretando que sería bueno per se, más no adecuado. Así, no se trataba de distribuir mejor los frutos del crecimiento, sino de crecer más para que todos recibieran una porción mayor que antes, pero manteniendo la misma proporción que les fuera otorgada por el sistema. Lo que ocurrió fue una concentración desproporcional de la riqueza en los estratos más altos, disminuyendo dramáticamente en los niveles más bajos (Cf: Max-Neef, 1986a).

Frente a lo planteado, la salida que propone es la búsqueda de la utopía que contemple una sociedad no sólo posible, sino deseable desde el punto de vista humanista, que haga visibles a los siempre excluidos de las políticas y las mediciones, una sociedad que rescate una dimensión social a escala humana, pues los seres humanos se desarrollan de acuerdo a las relaciones que mantiene con su entorno.

Todo tipo de entorno económico, espacial, político, cultural y natural, puede tener tanto una dimensión óptima como una dimensión crítica. Yo defino la primera como humanizadora y la segunda como alienante. En la primera los humanos son capaces de alcanzar un 
sentido de identidad e integración, mientras que en la segunda sólo pueden endosar a otros su integridad individual. Dentro de una, la persona percibe las consecuencias de lo que hace o decida por sí misma. En la otra, el ser humano se resigna a dejar que otros actúen y decidan por él. En la primera se hace posible el desarrollo de las personas; en la otra sólo el desarrollo de los objetos. El logro de un equilibrio dinámico entre naturaleza, seres humanos y tecnología (...) sólo es posible cuando los seres humanos tanto a nivel colectivo como individual, se sienten directamente responsables de las consecuencias de sus acciones dentro de su entorno y, a su vez, esto sólo es posible si las dimensiones de dicho entorno se mantienen a escala humana (Max-Neef et al., 1986b:152-153).

En este sentido, la concepción propuesta debe orientarse hacia la autodependencia, la satisfacción de las necesidades humanas y la articulación orgánica de los hombres con su entorno físico-social. Esta sólo sería posible a partir del protagonismo de la gente, desde donde se logre transformar a la persona-objeto en persona-sujeto del desarrollo y superar la aplicación de modelos gigantísticos jerárquicos de arriba hacia abajo; es decir, en el fondo, subyace un problema de escala (Max-Neef et al., 1986b).
Así, el desarrollo a escala humana, es definido a partir de los elementos antes señalados.

Tal desarrollo se concentra y sustenta en la satisfacción de las necesidades humanas fundamentales, en la generación de niveles crecientes de autodependencia y en la articulación orgánica de los seres humanos con la naturaleza y la tecnología, de los procesos globales con los comportamientos locales, de los personal con lo social, de la planificación con la autonomía y de la sociedad civil con el Estado (Max-Neff et al, 1986b:14).

\section{El desarrollo humano}

Otra respuesta a los modelos y concepciones economicistas que han dominado la escena mundial, proviene del Programa de las Naciones Unidas para el Desarrollo (PNUD) y su propuesta de Desarrollo Humano ${ }^{4}$.

En lo fundamental, dicha propuesta busca una óptica distinta en cuanto a la consideración de cuáles indicadores de desarrollo son los más apropiados para determinar la condición de un país y su consiguiente medición. Tradicionalmente, se ha considerado como variable el Producto Interno Bruto (PIB) -su crecimiento o disminución-, lo que ha llevado a una interpretación mecanicista que

4 Desde el año 1990, el Programa de las Naciones Unidas para el Desarrollo (PNUD) publica anualmente el Informe de Desarrollo Humano, que se ha constituido como alternativo a los Informes sobre Desarrollo Mundial que periódicamente realiza el Banco Mundial y cuya naturaleza es básicamente economicista. 
considera al aumento de los ingresos como sinónimo de desarrollo.

Así mismo, han hecho énfasis en los medios para alcanzar el desarrollo, considerando éstos como fines en sí mismo, lo que solapa el hecho de que el objetivo fundamental del desarrollo es beneficiar a la gente. Si bien no se discute la importancia y utilidad de las estadísticas acerca de los ingresos nacionales, las mismas no reflejan la composición ni los beneficiarios reales, además que los individuos valoran logros que no se materializan inmediatamente en términos de mayores ingresos o crecimiento. En otras palabras, no existe un vínculo automático entre el crecimiento del ingreso y el progreso humano (PNUD, 1990; PNUD-OCEI, 1998; PNUD-OCEI, 1999; PNUD-OCEI, 2001).

Dentro de esta perspectiva, para el PNUD el Desarrollo Humano significa tanto el proceso de ampliar las oportunidades de las personas como el nivel de bienestar que han alcanzado, distinguiéndose dos aspectos; por un lado, la formación de capacidades humanas para mejorar su condición y la forma cómo los individuos las emplean. Es decir, más que un modelo-que corren el peligro de convertirse en referencias indiscutibles que justifican actos de poder- el $\mathrm{DH}$ es considerado como un camino (PNUD, 1990; PNUD-OCEI, 1998; PNUD-OCEI, 2001).

En este sentido,

El Desarrollo Humano es un proceso mediante el cual se amplían las oportunidades de los individuos, las más importantes de la cuales son una vida prolongada y saludable, acceso a la educación y el disfrute de un nivel de vida de- cente. Otras oportunidades incluyen la libertad política, la garantía de los derechos humanos y el respeto a sí mismo (PNUD, 1990:33).

La idea que habita tras este concepto es que el cambio en las condiciones económicas y sociales es deseable únicamente si se mejora la calidad de vida, entendida ésta como ampliación de las oportunidades de las personas para decidir cómo vivir sus vidas. Así mismo, la idea de la libertad es básica para que las personas -individual y colectivamentepuedan desarrollar sus potencialidades y contar con oportunidades razonables para llevar una vida productiva y creativa conforme a sus necesidades e intereses (Cf: PNUD, 1990; PNUD-OCEI, 1998; PNUD-OCEI, 2001).

Oportunidades, capacidades, potencialidades y libertades son los argumentos teóricos sobre los cuales se construye el concepto de desarrollo humano, premisas éstas que toman del enfoque de desarrollo propuesto por Amartya Sen, que revisaremos en lo inmediato.

\section{El desarrollo como libertad o enfoque de capacidades}

La concepción del desarrollo como libertad, también conocido como enfoque de capacidades, es propuesta y sostenida por Amartya Sen, premio Novel de Economía 1998.

Este enfoque propone colocar el problema del desarrollo en los fines que lo hacen posible más que en los medios para alcanzarlos, por lo que centrar el punto de atención en las libertades humanas-la libertad como principal medio y fin del desarrollo- contrasta con las visiones 
que lo reducen al crecimiento del PIB, al aumento de las rentas personales, a la industrialización, al avance tecnológico o a la modernización social (Sen, 2000).

Para Sen (2000), la diferencia entre las dos perspectivas -la centrada en la riqueza económica y la centrada en la libertad de llevar la vida que podamos llevar- es importante en la conceptualización del desarrollo.

Si tenemos razones para querer más riqueza, hemos de preguntarnos ¿cuáles son esas razones, cómo actúan, de qué dependen y qué cosas podemos hacer con más riqueza? En realidad, generalmente tenemos excelentes razones para querer poseer más renta o más riqueza, y no es porque la renta y la riqueza sean deseables en sí mismas, sino porque, por norma, son admirables medios de uso general para tener más libertad con la que poder llevar el tipo de vida que tenemos razones para valorar. La utilidad de la riqueza reside en las cosas que nos permite hacer, es decir, en las libertades fundamentales que nos ayuda a conseguir (Sen, 2000: 30).

En la posición adoptada por Sen se desprenden dos aspectos claves en la concepción de libertad; por un lado los procesos que facilitan y hacen posible la libertad de acción, y por el otro las oportunidades reales que tienen los individuos de acuerdo a sus circunstancias sociales y personales.

La falta de libertad puede deberse a procesos inadecuados (como la violación de los derechos del voto o de otros derechos políticos o humanos) o a las insuficientes oportunidades que tienen algunas personas para conseguir lo que mínimamente les gustaría conseguir (incluida la falta de oportunidades tan elementales como la capacidad para escapar de una muerte prematura, de la morbilidad evitable o de la inanición involuntaria (Sen, 2000:34).

En este sentido, la expansión de las capacidades de las personas para llevar el tipo de vida que valoran y que tienen razones para valorar, es clave como concepto dentro de la propuesta. Se entiende por capacidad la habilidad de una persona para hacer actos valiosos o alcanzar estados para ser valiosos; éstas pueden aumentarse por políticas públicas, pero los individuos pueden influir en su rumbo haciendo uso eficaz de sus capacidades (por ejemplo, la participación) (Sen, 1998; 2000; 2001; 2004).

Ahora bien, el conjunto de capacidades de una persona representa las distintas combinaciones alternativas que ese sujeto puede hacer o ser, entendidas como funcionamientos (estados y acciones) que puede lograr. Estos funcionamientos, entendidos como partes del estado de una persona o las cosas que logra hacer o ser al vivir, reflejan una visión de la vida en tanto combinaciones de varios quehaceres y seres, donde el bienestar y la calidad de vida deben ser evaluados en términos de la capacidad para lograr funcionamientos valiosos (Sen, 1998; 2000; 2004).

Como se desprende de lo expuesto hasta ahora, en su propuesta Sen (1998; 2000; 2004) otorga especial importancia a la libertad individual, primero, por una razón de evaluación, y, segundo, por una razón de eficacia. La primera, en función de juzgar el éxito de una sociedad en tanto el disfrute o no, el aumento o disminución, de las libertades de que disponen 
sus miembros, pues es importante a la hora de realizar las cosas que tenemos razones para valorar y para determinar las ventajas individuales y el progreso social; es decir, de aumentar las oportunidades para obtener resultados valiosos. La segunda descansa en el hecho de que la libertad es determinante en la iniciativa individual y en la eficacia social, en tanto mejora la capacidad de los individuos tanto para actuar y ayudarse a sí mismo, como para influir en el mundo que los rodea.

La libertad individual es esencialmente un producto social, y existe una relación de doble sentido entre 1) los mecanismos sociales para expandir las libertades individuales y 2) el uso de las libertades individuales no sólo para mejorar las vidas respectivas sino también para conseguir que los mecanismos sociales sean mejores y más eficaces (...) Son los propios individuos los que deben asumir la responsabilidad del desarrollo y de la transformación del mundo en el que viven (...) Como seres humanos competentes, no podemos eludir la tarea de juzgar cómo son las cosas y qué es necesario hacer (...) Nuestro sentido de la responsabilidad no tiene por qué referirse sólo a las aflicciones que puede causar nuestra propia conducta (aunque eso también es muy importante), sino también, en términos más generales, a las miserias que observamos a nuestro alrededor y que está a nuestro alcance remediar (Sen, 2000:49, 338 y 339).

Este último punto guarda estrecha relación con el concepto de agente, entendido como la persona que actúa provocando cambios, y cuyos logros deben juzgarse en función de sus propios valores y objetivos, sean estos logros de bienestar (ayudarse a sí mismo) o logros de agente (influir en el mundo); es decir, el individuo como miembro del público y como participante en actividades económicas, sociales y políticas (Sen, 1998; 2004).

En base a estos argumentos, Sen considera que

El proceso de desarrollo puede considerarse como una ampliación de la libertad humana. El éxito de una economía y de una sociedad no puede separase de las vidas que pueden llevar los miembros de la sociedad. Puesto que no solamente valoramos el vivir bien y en forma satisfactoria, sino que también apreciamos el tener control sobre nuestras propias vidas, la calidad de la vida tiene que ser juzgada no solamente por la forma en que terminamos viviendo, sino también por las alternativas substanciales que tenemos (...) Ya que la evaluación de la libertad puede ser susceptible tanto a lo que hace una persona como a las alternativas que tiene, la libertad proporciona una perspectiva más amplia al juzgar la ventaja humana, y por medio de ella, evaluar el éxito social. Este es el razonamiento básico que proporciona la base para considerar el desarrollo como libertad (Sen, 1999: 1 y 2).

La importancia de esta visión reside en que vista la libertad como fin y medio del desarrollo, exige eliminar las principales fuentes de privación de la misma -la pobreza, la tiranía, la escasez de oportunidades económicas, las privaciones sociales, el abandono de los servicios públicos, el intervencionismo excesi- 
vo del Estado, la intolerancia de Gobiernos represivos, etc-. Así, las capacidades reflejan el poder de elegir y actuar de las personas, su libertad para hacer o ser, realizar sus metas, alcanzar su propio bienestar y lograr su calidad de vida, lo que significa una mayor potencialidad de la gente para mejorar su bienestar individual y colectivo por la vía de la ampliación de las opciones y una mayor libertad (Cf.: Sen, 1998; 2000; PNUD, 2001).

\section{A manera de cierre}

Como se desprende del recorrido teórico realizado, siempre se ha considerado la generación de riqueza y el crecimiento como el indicador fundamental de medición del desarrollo y, en consecuencia, como el elemento básico desde el cual se conceptualiza.

Sin embargo, existe suficiente evidencia empírica que refuta está creencia, aunque, sin dudas, es indiscutible su importancia como elemento para determinar el grado de desarrollo de una sociedad, lo que ha motivado preocupación y esfuerzos por tratar de encontrar definiciones alternativas que incluyan factores que contribuyan a enriquecer el concepto y que los integre estratégicamente -más que los excluya-, de manera de ampliar su alcance e impacto en la sociedad.

Es decir, además del crecimiento, incorporar su distribución y redistribución como mecanismo de equidad, el cuidado del medio ambiente, el fomento a la innovación y el progreso técnico, la satisfacción de las necesidades humanas espirituales y materiales, la promoción y creación de oportunidades sociales y la garantía de las libertades básicas que per- mitan fomentar las capacidades de los individuos, con el objetivo fundamental de mejorar la calidad de vida de las personas.

Nos atreveríamos a agregar dos elementos; primero, el de la responsabilidad -tanto individual como social-, que de garantía de construcción progresiva y corrección oportuna de las rutas estratégicas establecidas conceptualmente, $y$, segundo, la transparencia, que haga visible y claro el manejo material y social de la alternativa en edificación. Se considera que estos elementos respaldan el hecho mismo de la integración e integralidad de los factores, que implican una manera distinta de entender y enfocar el problema y de concebir los productos de políticas que resulten de ella.

La sola referencia de estos elementos da cuenta de la complejidad que rodea este proceso, lo que involucra una diversidad de actores que, más que oponerse, deben complementarse en la consecución de los objetivos y metas, por lo que la ruta de la discusión actual acerca de lo que se entiende por desarrollo, entrando el nuevo siglo, rebasa con creces los protagonismos que en antaño oponían al Estado y Mercado, pero también debe superar las opciones terceristas que integran ambos y adicionan a la sociedad civil sin tener claro el papel que deben cumplir de acuerdo el horizonte a alcanzar. Si bien es necesario alcanzar esta complementación, no es menos cierto que no existen recetas normativas para lograrlo.

Lo que sí está claro es que visto el desarrollo como proceso deliberado, los actores principales que lo impulsan -el Estado y la sociedad en general- deben 
compartir de manera consensuada el modelo societal al cual se aspira y su horizonte temporal para, a partir de allí, asumir una concepción que guíe a los individuos, hombres o ciudadanos en la búsqueda del bienestar personal y general.

Así mismo, es evidente que el desarrollo es, ante todo, un proceso altamente complejo, cuyos elementos están fuertemente interconectados, además de ser interdependientes unos con los otros. Habría que agregar, además, que una concepción compartida socialmente ayudaría a superar obstáculos que se creen insalvables y a innovar -puesto que las impactan-en la formación, formulación y ejecución de políticas públicas que garanticen su buena marcha y feliz término.

\section{Referencias bibliográfícas}

Anderson, Perry (2001) Historia y Lecciones del Neoliberalismo, en Houtart, Francois y Polet, Francois (Coord.) El Otro Davos. Globalización de Resistencias y de Luchas. Plaza y Valdés Editores. Primera Edic. 2000. Primera Reimpresión 2001. México. 181pp.

Banco Mundial (BM) (2000). En el Umbral del Siglo XXI. Informe Sobre el Desarrollo Mundial 1999-2000. Ediciones Mundi-Prensa. Madrid. 291 pp.

Betancourt Garcia, Mauricio (2004). Teorías y Enfoques del Desarrollo. Escuela Superior de Administración Pública. Programa de Administración Pública Territorial. Bogotá. 295pp.

Bustelo, Pablo (1999). Teorías Contemporáneas del Desarrollo Económico. Editorial Síntesis. Primera Edición, Primera Reimpresión. Madrid. 303pp.

Castellano Bohórquez, Hercilio (2005) La Planificación del Desarrollo Sostenible. Centro de Estudios del Desarrol- lo. Consejo Central de Estudios de Postgrado. Universidad Central de Venezuela. Caracas, 195 pp.

Central Latinoamericana de Trabajadores (CLAT) (1994). Neoliberalismo y Movimiento de los Trabajadores. Colección CLAT. Fondo Latinoamericano de Ediciones Sociales. Caracas. 475pp.

Comisión Económica Para América Latina y el Caribe (CEPAL). (1990). Transformación Productiva con Equidad. Santiago de Chile. Pp. 185.

Comisión Económica Para América Latina y el Caribe (CEPAL) (1991) EI Desarrollo Sustentable: Transformación Productiva, Equidad y Medio Ambiente. Mayo, Santiago de Chile. 154 pp. [Documento en Línea]. Disponible: http://www.eclac.cl. [Consulta: 2000, Mayo 28]

Comisión Económica Para América Latina y el Caribe (CEPAL) (1992) Educación y Conocimiento: Eje de la Transformación Productiva con Equidad. Agosto. Santiago de Chile. 270 pp. [Documento en Línea]. Disponible: http://www.eclac.cl. [Consulta: 2000, Mayo 28]

Comisión Económica Para América Latina y el Caribe (CEPAL). (1996). Equidad y Transformación Productiva: Un Enfoque Integrado. Santiago de Chile. Pp. 252.

Comisión Económica Para América Latina y el Caribe (CEPAL). (2000). Equidad, Desarrollo y Ciudadanía. Vigésimo Octavo Período de Sesiones. México, DF. [Documento en Línea]. Disponible: http://www.eclac.cl. [Consulta: 2000, Mayo 28]. Pp. 379

De La Peña, Sergio (1979). El Antidesarrollo de América Latina. Siglo XXI Editores. 7ma Edic. México. 205 pp.

De Lisio, Antonio (1999). Desarrollo Sustentable: Opciones y Limitaciones para 
América Latina y el Caribe. Cuadernos del CENDES. Año 16. $\mathrm{N}^{\circ} 42$. Segunda Época. Septiembre-Diciembre, pp 1-23.

Fajnzylber, Fernando (1983). La Industrialización Trunca de América Latina, México, D.F., Centro de Economía Transnacional/Editorial Nueva Imagen, $1983,416 p$

Fajnzylber, Fernando (1990). Industrialización en América Latina: de la "Caja Negra" al "Casillero Vacío". Cuadernos de la CEPAL. N 60. Santiago de Chile. Capítulo I. Pp 11-35.

Fernández-Muro, Clara (2004). La Macro Estructuralista y el Neoestructuralismo Latinoamericano desde 1983. Tema 17. Teorías del Desarrollo. Departamento de Economía Aplicada I. Facultad de Ciencias Económicas y Empresariales. Universidad Complutense de Madrid. 20 pp.

FURTADO, Celso (1993). La Cosmovisión de Prebisch, en IGLESIAS, Enrique V (Editor). El Legado de Raúl Prebisch. Banco Interamericano de Desarrollo. Washington, 204pp. Documento en Línea. Disponible: www. iadb.org/pub. Consulta: Marzo, 2000.

GABALDÓN, Arnoldo José (2006). Desarrollo Sustentable. La Salida de América Latina. Editorial Grijalbo. Corporación Andina de Fomento. Primera Edición. Caracas, 490 pp.

Gómez, Emeterio (1998). La Crisis de la Ciencia Económica. Edit. Panapo. Primera Edic. Caracas. 296pp.

Guillén R, Arturo (2006). Raul Prebisch, Crítico temprano del Modelo Neoliberal. Economía Unam. Vol. IV. Núm. 10. Documento en Línea. Disponible: www. ejournal.unam.mx-ecunamecunam 10-ECU000401006. Consulta: Nov. 2008

Iglesias, Enrique (1993). La Búsqueda de un Nuevo Consenso Económico en
América Latina, en IGLESIAS, Enrique $V$ (Editor). El Legado de Raúl Prebisch. Banco Interamericano de Desarrollo. Washington, 204pp. Documento en Línea. Disponible: www.iadb.org/pub. Consulta: Marzo, 2000.

Jiménez Herrero, Luis M (1997). Desarrollo Sostenible y Economía Ecológica. Integración Medio Ambiente-Desarrollo y Economía-Ecología. Editorial Síntesis. Economía. Serie Actualidad. Primera Edición. Primera Reimpresión. Madrid. 365 pp.

Max-Neef. Manfred (1986a). La Economía Descalza. Señales desde el Mundo Invisible. 1era Edic. en Inglés 1982. Edic. en Sueco 1984. Edic. en Español 1986. Traduc. Estela Lorca. Edit. Nordan. Buenos Aires, $249 \mathrm{Pp}$.

Max-Neef. Manfred; ELIZALDE, Antonio y HOPENHAYN, Martín (1986b). Desarrollo a Escala Humana: Una Opción para el Futuro. Fundación Hammarskjöld. Número Especial. S/E. S/F.

Maza Zavala, Domingo F (2006a). EIDesarrollo, Mito o Utopía, en Celso Furtado, Vigencia de un Pensador. Banco Central de Venezuela. Colección Científicos Sociales Contemporáneos. Primera Edición. Caracas, $117 \mathrm{pp}$.

Maza Zavala, Domingo F (2006b). Conciencia Latinoamericana: El aporte de Raúl Prebisch. Raúl Prebisch. Pensamiento Renovador. Banco Central de Venezuela. Colección Científicos Sociales Contemporáneos. Primera Edición. Caracas, 151 pp.

Olivé, Iliana (2004). Macroeconomía Estructuralista y Neoestructuralismo Latinoamericano. Tema 15. Teorías del Desarrollo. Departamento de Economía Aplicada I. Facultad de Ciencias Económicas y Empresariales. Universidad Complutense de Madrid. 19 pp. 
Pirela, Arnoldo (1990). La Escuela latinoamericana de Pensamiento Económico Social. Publicaciones CENDES. Colección Jorge Ahumada. Vadell Hermanos Editores. Primera Edición. Caracas, $171 \mathrm{pp}$.

Programa de las Naciones Unidas para el Desarrollo (PNUD) (1990). Desarrollo Humano. Informe 1990. Tercer Mundo Editores. Primera Edición en Español. Traduc. Ángela García. PNUDOxford University Press. Bogotá, 240 pp.

Programa de las Naciones Unidas para el Desarrollo (PNUD). Oficina Central de Estadística e Informática (OCEI). (1998). Informe sobre Índice y Entorno de Desarrollo Humano en Venezuela 1998. S/Edit. S/Edic. Caracas, $194 \mathrm{pp}$.

Programa de las Naciones Unidas para el Desarrollo (PNUD). Oficina Central de Estadística e Informática (OCEI). (1999). Informe sobre Índice y Entorno de Desarrollo Humano en Venezuela 1999. CDB Publicaciones. Primera Edición. Caracas, 211 pp.

Programa de las Naciones Unidas para el Desarrollo (PNUD). Oficina Central de Estadística e Informática (OCEI). (2001). Informe sobre Desarrollo Humano en Venezuela, 2000. Caminos para Superar la Pobreza. CDB Publicaciones. 1era Edic. 214 $\mathrm{pp}$.

Ramos, Joseph y SUNKEL, Osvaldo (1995). Introducción: Hacia una Síntesis Neoestructuralista. En SUNKEL, Osvaldo (Compilador). El Desarrollo Desde Dentro. Un Enfoque Neoestructuralista para la América Latina. CEPAL-Fondo de Cultura Económica. Serie Lecturas. Primera Edición 1991. Primera Reimpresión 1995. Santiago de Chile. Pp. 15-32.
Reed, David (Editor) (1996). Ajuste Estructural, Ambiente y Desarrollo Sostenible. CENDES, WWF, Nueva Sociedad. 1era Edic. $380 \mathrm{Pp}$.

Sen, Amartya (1998). Capacidad y Bienestar, en NUSSBAUM, Martha y SEN Amartya (Comp.). La Calidad de Vida. Fondo de Cultura Económica. The United Nations University. 1 ra Reimpresión. México. Pp 54-83.

Sen, Amartya (1999). Romper el Ciclo de la Pobreza: Invertir en la Infancia. Conferencia Magistral. BID. Departamento de Desarrollo Sostenible. División de Desarrollo Social. [Documento en Línea]. Disponible: http://www. iadb.org [Consulta: Octubre, 2000]

Sen, Amartya (2000). Desarrollo y Libertad. Edit. Planeta. Primera Edic. Mayo. Traduc. Esther Rabasco y Luis Toharia. España. 423 pp.

Sen, Amartya (2001). El Nivel de Vida. Editorial Complutense. Primera Edición. Quinta Reimpresión. Traducción: José Miguel Parra Ortiz y María Elisa González González. Madrid. 180 pp.

Sen, Amartya (2004) Nuevo examen de la Desigualdad. Alianza Editorial. Ciencias Sociales. Primera Edición. Tercera Reimpresión. Traducción: Ana María Bravo. Revisión: Pedro Schwartz. Madrid. $221 \mathrm{pp}$.

Sunkel, Osvaldo y PAZ, Pedro (1977). El Subdesarrollo Latinoamericano y la Teoría del Desarrollo. Siglo XXI Editores. 13va Edic. Caracas. 385pp.

Sunkel, Osvaldo (1994). La Crisis Social de América Latina: Una Perspectiva Neoestructuralista. En CONTRERAS QUINA, Carlos (Compilador). EI Desarrollo Social. Tarea de Todos. Comisión Sudamericana de Paz, Seguridad y Democracia. Editorial Nueva Sociedad. Primera Edición. Caracas. Pp. 27-93. 
Sunkel, Osvaldo (1995). Del Desarrollo Hacia Adentro al Desarrollo Desde Dentro. En Sunkel, Osvaldo (Compilador). EI Desarrollo Desde Dentro. Un Enfoque Neoestructuralista para la América Latina. CEPAL-Fondo de Cultura Económica. Serie Lecturas.
Primera Edición 1991. Primera Reimpresión 1995. Santiago de Chile. Pp. $35-80$.

Torres Oliveros, Miguel (2006). Fernando Fajnzylber: Una Visión Renovada del Desarrollo de América Latina. CEPAL. Santiago de Chile. 417 pp. 